\title{
Effective Inflow Conditions for Turbulence Models in Aerodynamic Calculations
}

\author{
Philippe R. Spalart * \\ Boeing Commercial Airplanes, P.O. Box 3707, Seattle, WA 98124, USA \\ Christopher L. Rumsey ${ }^{\dagger}$ \\ NASA Langley Research Center, Hampton, VA 23681-2199, USA
}

First-author contact information:

Phone: (425) 2341136

Fax: (425) 2378281

e-mail: philippe.r.spalart@boeing.com

Keywords: CFD, turbulence modeling, boundary conditions

\begin{abstract}
The selection of inflow values at boundaries far upstream of an aircraft is considered, for one- and twoequation turbulence models. Inflow values are distinguished from the ambient values near the aircraft, which may be much smaller. Ambient values should be selected first, and inflow values that will lead to them after the decay second; this is not always possible, especially for the time scale. The two-equation decay during the approach to the aircraft is shown; often, the time scale has been set too short for this decay to be calculated accurately on typical grids. A simple remedy for both issues is to impose floor values for the turbulence variables, outside the viscous sublayer, and it is argued that overriding the equations in this manner is physically justified. Selecting laminar ambient values is easy, if the boundary layers are to be tripped, but a more common practice is to seek ambient values that will cause immediate transition in boundary layers. This opens up a wide range of values, and selection criteria are discussed. The turbulent Reynolds number, or ratio of eddy viscosity to laminar viscosity has a huge dynamic range that makes it unwieldy; it has been widely mis-used, particularly by codes that set upper limits on it. The value of turbulent kinetic energy in a wind tunnel or the atmosphere is also of dubious value as an input to the model. Concretely, the ambient eddy viscosity must be small enough to preserve potential cores in small geometry features, such as flap gaps. The ambient frequency scale should also be small enough, compared with shear rates in the boundary layer. Specific values are recommended and demonstrated for airfoil flows.
\end{abstract}

\section{Introduction}

Turbulence models based on transport equations are now omnipresent, but the CFD community's knowledge of appropriate boundary conditions for the turbulence variables at distant boundaries is incomplete. There is much room for clarity and helpfulness in some of the original turbulence-model papers, and in the manuals and Graphical User Interfaces of widely-used codes. Solid physical interpretations of the values taken by the primary variables and the eddy viscosity derived from them are needed. A contributing factor is the large dynamic range of the variables; for instance, the eddy viscosity may logically be compared with the molecular viscosity or with the product of velocity and length of the aircraft, and these two numbers may differ by a factor of $10^{8}$, the Reynolds number. The choice between these two scales is a source of confusion, and interferes with the establishment of guidelines. This situation creates pitfalls, among them failures to produce turbulence where intended by the user, ${ }^{1}$ and excessive eddy-viscosity values in regions which have not produced turbulence and should remain irrotational. These pitfalls, which are preventable,

\footnotetext{
*Boeing Senior Technical Fellow, Member AIAA, Mail Stop 67-LM.

${ }^{\dagger}$ Senior Research Scientist, Member AIAA, Computational Aerosciences Branch, Mail Stop 128.
} 
may well go undetected when the models are treated as black boxes. Our purpose here is to recommend effective prevention measures. "Effective" refers to boundary-condition practices which do not add much to the users' burden, and ensure a low sensitivity of the results to trivial parameters. For instance, it would be unacceptable to get different results from a computation with a domain 50 chords in radius, and another domain 60 chords in radius. Progress in science and engineering depends on repeatability, and on building an experience base that is genuine.

It may help to start from the "ideal situation" a trustful CFD user would expect. The incoming stream has welldefined turbulence kinetic energy and dissipation, $k$ and $\varepsilon$, and eddy viscosity derived from them, $\nu_{t}=C_{\mu} k^{2} / \varepsilon$ (for a physical reason and not only dimensional analysis). These quantities correctly respond to velocity gradients while approaching the aircraft, and couple meaningfully with the boundary-layer turbulence. It is simply a matter of knowing $k$ and $\varepsilon$ far upstream, preferably from measurements. These values are "realistic."

This vision fails, primarily because describing all of turbulence with two numbers is insufficient. Here, we disregard internal flows and in particular gas-turbine applications, for which the coupling with ambient turbulence is much stronger, and address only external aircraft flows. In the atmospheric boundary layer, modeled with Reynolds-averaged Navier-Stokes (RANS) equations, the eddy viscosity on a windy day can well take values such as $50 \mathrm{~m}^{2} / \mathrm{s}$, and the chord Reynolds number of a large airplane based on this eddy viscosity would be near 5 . This is untenable, of course. The reason is that the eddies which may couple with the boundary layer have sizes of the order of $1 \mathrm{~cm}$, rather than $100 \mathrm{~m}$, so that only a tiny fraction of the "atmospheric" $k$ is relevant (the analogous estimates in a wind tunnel are not as immensely different, but the physical consequences are the same). This relevant fraction is deduced from the atmospheric dissipation rate, much better than from the atmospheric $k$, and it is not sharply defined. Moreover, it has no reason to obey the decay equations used to calibrate two-equation models in isotropic turbulence. In reality, the kinetic energy relevant to the aircraft flow varies very little over the size of the CFD domain. This fact is in strong conflict with common practices in CFD, since inflow values of $\varepsilon / k$ near $U_{\infty} / c$ are routinely used, where $U_{\infty}$ is the speed of the aircraft and $c$ the wing chord. Over an approach to the aircraft of length $50 c, k$ then drops by a factor of about 75 , if the solution is accurate according to the decay equations. This is obviously unrealistic behavior, accepted in an attempt to obtain the desired behavior in the boundary layers. Furthermore, only the eddy viscosity has a strong influence on the flow field, at least at moderate Mach numbers; $k$ and $\varepsilon$ separately do not. In fact, most CFD users are only interested in whether the solution is laminar or turbulent, and of those, the vast majority have no interest in laminar regions. Fully-Turbulent (FT) behavior is safer and gives better convergence, and can be viewed as the standard mode of use of RANS models today.

In addition to being unrealistic, the precipitous decay associated with such inflow values is not calculated accurately. A typical grid spacing near the inflow boundary is larger than $c$, which is too large to resolve a decay at a rate $1 / c$. This is observed in otherwise fully normal CFD solutions; the decay is grossly under-estimated and griddependent, as will be shown below. The boundary-layer turbulence responds only to what we will call the ambient values of the turbulence variables: those in the vicinity of the aircraft. Thus, for example, grid-dependent ambient values can have an influence on the location in the boundary layer where the model trips to turbulence. Because small variations in transition location do not typically have a glaring impact on the global results, this problem is largely out of sight for the user. However, the situation is clearly problematic from the point of view of consistency.

Most CFD codes handle the issue of freestream turbulence differently. There does not appear to be a consistent standard practice. Many codes assign default values and allow the user to override if desired. However, there is usually very little guidance given as to how different choices affect the decay rates and the resulting levels that are actually seen near the body. Some codes (such as the CFD++ $\operatorname{code}^{2}$ ) allow the user to disable the decay in some regions, which are manually defined with controls such as $x_{\min }$ and $x_{\max }$. This manner of control is not practical for reallife geometries. Other groups prevent the decay by imposing "floor values:" the turbulence variables are limited by non-zero minimum values. This limiting may conflict with the wall boundary condition for $k$, but this can be resolved using the SST model's $F_{1}$ function, ${ }^{3}$ for instance. More important is the question of whether violating the celebrated $k-\varepsilon$ equations by imposing floor values can be justified at all; this is taken up below.

In the remainder of the paper, most of the focus will be on the two-equation $k-\omega \mathrm{SST}$ model ${ }^{3}$ and the one-equation model of Spalart \& Allmaras (S-A), ${ }^{4}$ but theoretical aspects of the $k-\varepsilon$ model will also be considered. The equations for free decay are presented first, leading to "reverse-engineering" inflow values from ambient values; constrained decay is then examined. Then, the valid criteria that exist to pick ambient and/or inflow values are discussed. This is followed by examples which support the recommendations made, and a summary. 


\section{Decay Equations and Control}

\section{A. Free Decay}

Free decay rates of turbulence quantities can be very large and "non-physical" when commonly-used inflow values are specified. This can be seen from the solution to the $k-\varepsilon$ equations in the approaching flow field. A typical form of the complete $k-\varepsilon$ equations can be written:

$$
\begin{aligned}
\frac{D k}{D t} & =\mathcal{P}-\varepsilon+\frac{\partial}{\partial x_{j}}\left[\left(\nu+\frac{\nu_{t}}{\sigma_{k}}\right) \frac{\partial k}{\partial x_{j}}\right] \\
\frac{D \varepsilon}{D t} & =\frac{\varepsilon}{k}\left(C_{\varepsilon 1} \mathcal{P}-C_{\varepsilon 2} \varepsilon\right)+\frac{\partial}{\partial x_{j}}\left[\left(\nu+\frac{\nu_{t}}{\sigma_{\varepsilon}}\right) \frac{\partial \varepsilon}{\partial x_{j}}\right]
\end{aligned}
$$

with $\nu_{t}=C_{\mu} k^{2} / \varepsilon$, and $C_{\mu}=0.09$. In the approach to the aircraft, only the destruction terms are active, so that $U k_{x}=-\varepsilon$ and $U \varepsilon_{x}=-C_{\varepsilon 2} \varepsilon^{2} / k$ to a very good approximation. The solution is simple and linear for $k / \varepsilon$ :

$$
\frac{k}{\varepsilon}=\left(\frac{k}{\varepsilon}\right)_{F S}+\left(C_{\varepsilon 2}-1\right) \frac{x}{U}
$$

where the subscript $F S$ means "free-stream", or "inflow", and $x$ is the distance from the inflow to the field point. Control over the time scale $k / \varepsilon$ through the inflow value is difficult; nothing can prevent it from growing. Typically, $C_{\varepsilon 2}=1.92$.

The solutions for $k, \varepsilon$, and $\nu_{t}$ are then:

$$
\begin{aligned}
k & =k_{F S}\left[1+\left(C_{\varepsilon 2}-1\right)\left(\frac{\varepsilon}{k}\right)_{F S} \frac{x}{U}\right]^{\frac{-1}{C_{\varepsilon 2}-1}} \\
\varepsilon & =\varepsilon_{F S}\left[1+\left(C_{\varepsilon 2}-1\right)\left(\frac{\varepsilon}{k}\right)_{F S} \frac{x}{U}\right]^{\frac{-C_{\varepsilon 2}}{C_{\varepsilon 2}-1}} \\
\nu_{t} & =\nu_{t F S}\left[1+\left(C_{\varepsilon 2}-1\right)\left(\frac{\varepsilon}{k}\right)_{F S} \frac{x}{U}\right]^{\frac{C_{\varepsilon 2}-2}{C_{\varepsilon 2}-1}}
\end{aligned}
$$

It is seen that $\nu_{t}$ decays much more slowly than $k$ and $\varepsilon$ : the power is about -0.09 versus -1.1 and -2.1 , respectively. Numerical tests have confirmed this decay, but have also shown that it is calculated accurately from the onset only if $(\varepsilon / k)$ is small enough compared with $U_{\infty} / \Delta x$, where $\Delta x$ is the streamwise grid spacing. When $\Delta x$ is too large to support accurate computations in the far field, the decay simply tends to be delayed, and during the approach, both $(\varepsilon / k)$ and $U_{\infty} / \Delta x$ evolve in the direction needed to improve their ratio. This situation remains aesthetically undesirable, and likely to cause jumps during grid sequencing, among other things. It could also disturb the order of accuracy of the solver.

The freestream decay of $\omega$ (for the $k-\omega$ model) can be found in a similar fashion. For example, the $k-\omega$ SST model is given by:

$$
\begin{aligned}
\frac{D k}{D t} & =\mathcal{P}-\beta^{*} \omega k+\frac{\partial}{\partial x_{j}}\left[\left(\nu+\frac{\nu_{t}}{\sigma_{k}}\right) \frac{\partial k}{\partial x_{j}}\right] \\
\frac{D \omega}{D t} & =\frac{\gamma}{\mu_{t}} \mathcal{P}-\beta \omega^{2}+\frac{\partial}{\partial x_{j}}\left[\left(\nu+\frac{\nu_{t}}{\sigma_{\omega}}\right) \frac{\partial \omega}{\partial x_{j}}\right]+2\left(1-F_{1}\right) \frac{1}{\sigma_{\omega 2} \omega} \frac{\partial k}{\partial x_{j}} \frac{\partial \omega}{\partial x_{j}}
\end{aligned}
$$

where $\nu_{t}=a_{1} k / \max \left(a_{1} \omega, \Omega F_{2}\right), a_{1}=0.31, \Omega$ is the magnitude of vorticity, and $F_{1}$ and $F_{2}$ are blending functions. Here we use the standard convention that $\omega \equiv \varepsilon /\left(C_{\mu} k\right)$. The decay of $\omega$ in the freestream is:

$$
\omega=\omega_{F S}\left(1+\omega_{F S} \beta x / U\right) .^{-1}
$$

Because $\beta$ is equivalent to $C_{\mu}\left(C_{\varepsilon 2}-1\right)$ (with $C_{\mu}=\beta^{*}=0.09$ ), Eq. (9) is equivalent to Eq. (3), which can also be written as $1 / \omega=1 / \omega_{F S}+C_{\mu}\left(C_{\varepsilon 2}-1\right) x / U$.

Equations (4 - 6) and (9) are available to produce the inflow values needed, once ambient values have been chosen, or else to indicate that a set of ambient values is unreachable, with (3) expressing the dominant constraint.

Clearly, it is seen from these equations that the chosen freestream values of the turbulence quantities have a strong influence on the rate of decay of the turbulence values. Table 1 shows examples of typical freestream levels (for 
Table 1. Examples of effect of freestream levels on turbulence decay after $x / L=50$ (for $R e_{L}=10$ million)

\begin{tabular}{|cc|ccc|cccc|}
\hline$T u, \%$ & $\frac{\nu_{t, F S}}{\nu}$ & $\frac{k_{F S}}{U^{2}}$ & $\frac{\varepsilon_{F S} L}{U^{3}}$ & $\frac{\omega_{F S} L}{U}$ & $\frac{\nu_{t}}{\nu_{t, F S}}$ & $\frac{k}{k_{F S}}$ & $\frac{\varepsilon}{\varepsilon_{F S}}$ & $\frac{\omega}{\omega_{F S}}$ \\
\hline 0.001 & 0.01 & $1.5 \times 10^{-10}$ & $2.025 \times 10^{-12}$ & 0.15 & 0.96 & 0.59 & 0.37 & 0.62 \\
0.01 & 0.01 & $1.5 \times 10^{-8}$ & $2.025 \times 10^{-8}$ & 15 & 0.70 & 0.11 & $1.8 \times 10^{-4}$ & 0.016 \\
0.1 & 0.01 & $1.5 \times 10^{-6}$ & $2.025 \times 10^{-4}$ & 1500 & 0.47 & $7.5 \times 10^{-5}$ & $1.2 \times 10^{-8}$ & $1.6 \times 10^{-4}$ \\
1.0 & 0.01 & $1.5 \times 10^{-4}$ & $2.025 \times 10^{0}$ & $1.5 \times 10^{5}$ & 0.31 & $5.1 \times 10^{-7}$ & $8.1 \times 10^{-13}$ & $1.6 \times 10^{-6}$ \\
0.1 & 0.1 & $1.5 \times 10^{-6}$ & $2.025 \times 10^{-5}$ & 150 & 0.57 & $9.2 \times 10^{-4}$ & $1.5 \times 10^{-6}$ & $1.6 \times 10^{-3}$ \\
0.1 & 1.0 & $1.5 \times 10^{-6}$ & $2.025 \times 10^{-6}$ & 15 & 0.70 & 0.011 & $1.8 \times 10^{-4}$ & 0.016 \\
0.1 & 10 & $1.5 \times 10^{-6}$ & $2.025 \times 10^{-7}$ & 1.5 & 0.84 & 0.12 & 0.016 & 0.14 \\
0.1 & 100 & $1.5 \times 10^{-6}$ & $2.025 \times 10^{-8}$ & 0.15 & 0.96 & 0.59 & 0.37 & 0.62 \\
\hline
\end{tabular}

\begin{tabular}{|ccccc|}
\hline$\frac{\nu_{t}}{\nu}$ & $\frac{\nu_{t}}{U L}$ & $\frac{k}{U^{2}}$ & $\frac{\varepsilon L}{U^{3}}$ & $\frac{\omega L}{U}$ \\
\hline 0.0096 & $9.6 \times 10^{-10}$ & $8.9 \times 10^{-9}$ & $7.5 \times 10^{-13}$ & 0.09 \\
0.007 & $7 . \times 10^{-10}$ & $1.7 \times 10^{-9}$ & $3.6 \times 10^{-12}$ & 0.24 \\
0.0047 & $4.7 \times 10^{-10}$ & $1.1 \times 10^{-10}$ & $2.4 \times 10^{-12}$ & 0.24 \\
0.0031 & $2.1 \times 10^{-10}$ & $7.7 \times 10^{-11}$ & $1.6 \times 10^{-12}$ & 0.24 \\
0.057 & $5.7 \times 10^{-9}$ & $1.4 \times 10^{-9}$ & $3 \times 10^{-11}$ & 0.24 \\
0.7 & $7 . \times 10^{-8}$ & $1.7 \times 10^{-8}$ & $3.6 \times 10^{-10}$ & 0.24 \\
8.4 & $8.4 \times 10^{-7}$ & $1.8 \times 10^{-7}$ & $3.2 \times 10^{-9}$ & 0.21 \\
96 & $9.6 \times 10^{-6}$ & $8.9 \times 10^{-7}$ & $7.4 \times 10^{-9}$ & 0.09 \\
\hline
\end{tabular}

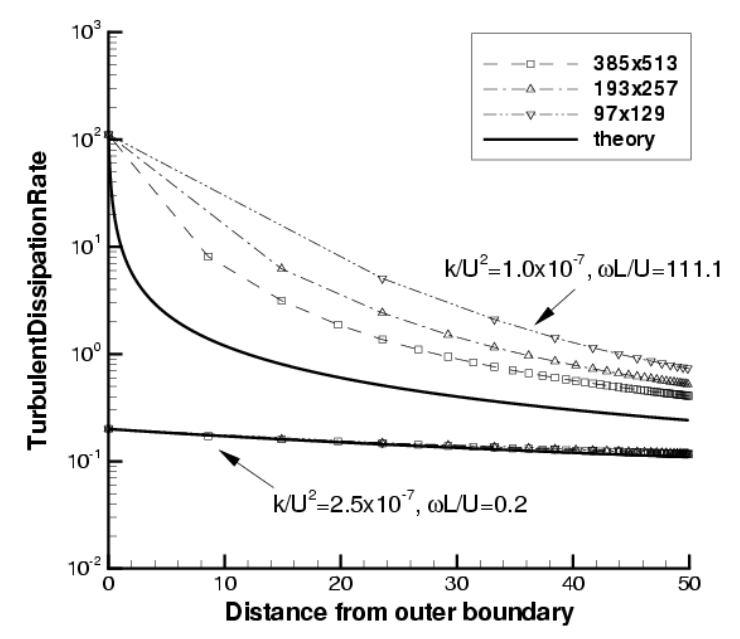

Figure 1. Evolution of $\omega L / U$ ahead of NACA 0012 airfoil, with $R e_{c}=10^{7}$. 
$R e_{L}=10 \times 10^{6}$ ), along with their decayed levels according to Eqs. (4 - 6) and (9) at a distance of $x / L=50$ downstream. The freestream turbulence intensity, in percent, is related to turbulent kinetic energy by the relation $T u=100 \sqrt{2 k /\left(3 U^{2}\right)}$. The highest value $\omega L / U$ can reach is 0.2415 ; many of the inflow-value sets chosen lead to 0.24 , in other words, $\omega_{F S}$ was set so high it lost control of the decayed value. The dramatic decay of $k$ and $\varepsilon$ is evident in some of these examples. Taking $T u$ and $\nu_{t, F S}$ as the parameters easiest to relate to and varying them independently, this table demonstrates that the higher the freestream turbulence intensity $(T u)$, the more rapid the decay; and the lower the freestream eddy viscosity level $\left(\nu_{t}\right)$, the more rapid the decay. In other words, to achieve "reasonable" decay rates, one is forced to use either very low levels of freestream $T u$ or very high levels of freestream $\nu_{t}$.

As mentioned earlier, for large decay rates, the coarseness of the grid far from the body may make the solver unable to support an accurate computation and the decay can be grossly underestimated. The behavior of $\omega$ for two different freestream turbulence levels is shown in Fig. 1. In particular, it is shown that for the higher freestream decay conditions, the computed results display a very noticeable grid dependence, only slowly approaching the correct answer as the grid is refined (upper curves). Using significantly lower values for freestream $\omega$, however, the decay rate is dramatically reduced, and CFD predicts the levels with greater precision on all three grids (lower curves).

The equivalent situation for the S-A model is most simple; in the absence of significant destruction term (which is discussed below) and of vorticity (which sets production), the transported variable $\tilde{\nu}$ and the eddy viscosity $\nu_{t}$ are uniform. The objection sometimes made to the absence of a destruction term, which may appear unphysical, is here a definite advantage. A trivial issue which nevertheless causes confusion is that some user interfaces do not make it clear whether $\tilde{\nu}$ or $\nu_{t}$ is being set; unfortunately, this is pivotal when the values are of the same order as the molecular viscosity $\nu$. An obliquely related issue is that several groups have used for inflow the value $\tilde{\nu}=1.34$, which gives $\nu_{t} / \nu=0.009$; this comes from a historical detail of the Baldwin-Barth model, ${ }^{5}$ using the round number $R e_{t}=k^{2} / \nu \varepsilon=0.1$. Unfortunately, this value is not very far from the boundary $\tilde{\nu} / \nu=\sqrt{\log \left(c_{t 3}\right) / c_{t 4}}$ between those solutions that are attracted by 0 once the production term becomes active, due to the $f_{t 2}$ function in the model, and those that are not. ${ }^{1}$ This boundary is at $\tilde{\nu} / \nu=0.60$ in the more common Version $1 \mathrm{a},{ }^{4}$ and at $\tilde{\nu} / \nu=0.22$ in Version 1 (AIAA paper 92-0439). The destruction term can steer $\tilde{\nu}$ into the basin of attraction, with unpredictable results. Values of $\tilde{\nu} / \nu$ in the 3-to-5 range are much more reliable when fully-turbulent behavior is desired.

\section{B. Constrained Decay}

The concept of constrained decay - or "floor values" - is simple: $k$ and $\varepsilon$ (or $\omega$ ) are not allowed to drop below some pre-set values. This method is used in the NASA PAB3D code, ${ }^{6}$ and was suggested to us by Dr. Abdol-Hamid (2006). For the S-A model, this technique is not necessary.

As mentioned earlier, the conflict with the wall boundary condition $k=0$ can be removed by conditioning the limiters on $F_{1}$ from the SST model, or a similar quantity in other models that detects the interior of the boundary layer. This conditioning should also prevent potential problems for cases with boundary layer relaminarization. A secondary issue is that preventing the free decay with an over-ride can create the appearance of non-convergence of the solution, since the residuals will remain non-zero; again, it is remediable and only a reporting issue.

The objection against disabling the classical $k-\varepsilon$ equations is deeper, but the discussion above regarding the actual behavior of that part of the turbulence that is of small scale compared with the atmospheric scales or even the wind-tunnel size is founded. This small-scale component of a larger turbulence field simply does not decay the way turbulence that has all its energy in the small scales would. Essentially, it has a supply of energy in the Kolmogorov cascade, and does not decay. A separate argument is that the physical merit of any influence the ambient values have inside the boundary layers via the model's diffusion terms is very debatable. In fact, this influence has at times been grievously troublesome, in the case of the pure $k$ - $\omega$ model, and of the Baldwin-Barth model. See Menter. ${ }^{7,8}$

A milder version of this "re-interpretation" of $k$ and $\varepsilon$ as not representing the totality of the turbulence occurs in the logarithmic layer. The two equations demand a plateau with $k^{+}=1 / \sqrt{C_{\mu}}$, yet the true TKE found from experiments and DNS is far from uniform, leading to the view that the modeled $k$ represents only the "active" energy, which has length scales of the order of the wall distance and contributes to the shear stress. In that sense, it is also a high-pass filtered version of the complete $k$. The description of the $k-\omega$ model by Wilcox ${ }^{9}$ also hints at such a view; see also $v^{\prime 2}$ in the $v^{2}-f$ model. ${ }^{10}$

At first sight, setting floor values adds to the user's burden; but in fact, these levels can be identical to the inflow values, and they become the ambient values. Thus, the situation is markedly simplified, and this option is practical. We can now turn to the choice of these ambient values. 


\section{Ambient Values}

Turbulence modeling is a pragmatic endeavor, and we are seeking "effective" values in the sense outlined above, without reference to "true" measurable levels of $k$ and $\varepsilon$, let alone $\nu_{t}$. We also consider the common situation in which the ambient values are picked to trigger turbulence in all boundary layers without local tripping, except perhaps at very low Reynolds numbers. Before making our recommendations, we review the possible bases for picking ambient values.

\section{A. Turbulent Reynolds Number}

It is natural to compare the eddy viscosity $\nu_{t}$ to the molecular viscosity $\nu$, but serious mis-conceptions have ensued. First, the idea that outside boundary layers it must satisfy $\nu_{t} / \nu \ll 1$, "since there is no turbulence," is erroneous as will be shown. Values much higher than this remain "effective," when the flow Reynolds number is high enough, unless of course laminar boundary layers are desired. Second, objections to very high values of $\nu_{t} / \nu$ are also erroneous, and have caused trouble in at least one code. The ratio $\nu_{t} / \nu$ had been limited to $10^{5}$, at first sight a very large number, in response to some convergence troubles; however, $10^{5}$ is routinely exceeded in jets at full-scale Aerospace Reynolds numbers. Any such hard-wired limiters should be removed from all codes. They create a Reynolds-number dependence that runs completely against turbulence theory in free shear flows. This also applies to limiters offered to users in some codes. In some situations, RANS models err by having too high an eddy viscosity; examples include free vortices, for most models, and fully-developed round jets, for the S-A model. A more accurate solution can then be obtained by manually lowering the eddy viscosity, and some users experiment, say with a limit such as $\tilde{\nu} / \nu \leq 10^{4}$. The danger is that in free shear flows, this limiter should scale proportionally to the flow Reynolds number, and the user's manuals give no guidance on this. Massive inaccuracies can again result at full-size Reynolds numbers.

The area in which $\nu_{t} / \nu$ has control and is meaningfully compared with unity concerns transition in the S-A model. Ambient values of $\tilde{\nu} / \nu$ well below 1 , such as 0.1 , will cause the model to remain dormant. Values well above 1 , such as 3, will cause it to grow turbulent in all boundary layers at reasonably high Reynolds numbers. This is by design. There is no reason to pick values much above 3, even when fully-turbulent behavior is desired. This will ensure that ambient values do not influence the interior of the boundary layer, except for steering it towards laminar or turbulent levels, which is binary.

\section{B. Boundary-Layer Levels, and Device Reynolds Numbers}

If the ambient values, which have been seen to be quite arbitrary, are not to influence the boundary layer through diffusion, these values need to be decidedly lower than values in the boundary-layer's outer region. These are of the order of $10^{-3} U^{2}$ for $k$, where $U$ is a typical edge velocity, and $10^{-4} U^{3} / \delta$ for $\varepsilon$, where $\delta$ is the boundary-layer thickness; finally, $\omega$ is simply of the order of $U / \delta$, and $\nu_{t}$ of the order of $10^{-3} U \delta$. It must be remembered that $\nu_{t}$ itself is not diffusing in two-equation models, and its ambient value is not directly relevant: the $k-\varepsilon$ equations tend to draw $\nu_{t}$ down near the boundary-layer edge. ${ }^{11}$ However, if floor values constrain the primary variables, they also constrain $\nu_{t}$.

An example of the corruption of the solution in the boundary layer by high ambient turbulence is shown in Fig. 2 . Here, the floor value for $k / U^{2}$ is $1.5 \times 10^{-6}$, and the floor value for $\omega L / U$ is varied. When it is very high (over 1000), the natural decay of $\omega$ is interrupted only $1 / 3$ of the way up the boundary layer, and the velocity profile gets damaged as a result. This illustrates a danger of the practice of imposing floor values: recall that with free decay, $\omega L / U$ could not exceed 0.24 . This figure, incidentally, confirms the estimate of $U / \delta$ for the outer-layer levels of $\omega$. Also note the large dynamic range of $\omega$, and therefore $\varepsilon$, within a boundary layer; wall values would not be relevant in this context.

Another very concrete consideration in aerospace applications is the gap regions between different elements of a wing. These are normally designed to allow a "potential core" between the two boundary layers. It is all too easy to set the ambient $\nu_{t}$ so high that this potential core is smeared, and to be ignorant of this fact, deeply hidden in the flow field. Yet, this will make CFD inaccurate, and sensitive to ambient values. The gap $d$ is often of the order of $1 \%$ of the chord, and the gap/eddy-viscosity Reynolds number $d U / \nu_{t}$ must be in excess of about 100, and therefore the chord/eddy-viscosity Reynolds number must be in excess of $10^{4}$. Even single-element wings have small features for which the potential flow should not be corrupted; the leading-edge radius is a few percent of the chord. Again, only extensive examinations of the flow field would exhibit such violations, so it is highly desirable to prevent them from ever occurring in the first place by good standard practices. Normally, the constraint for the eddy viscosity to be dominated by the boundary-layer eddy viscosity $\nu_{t}<10^{-3} U \delta$ is stronger than the gap constraint $\nu_{t} \ll U d$, but both should be kept in mind. 

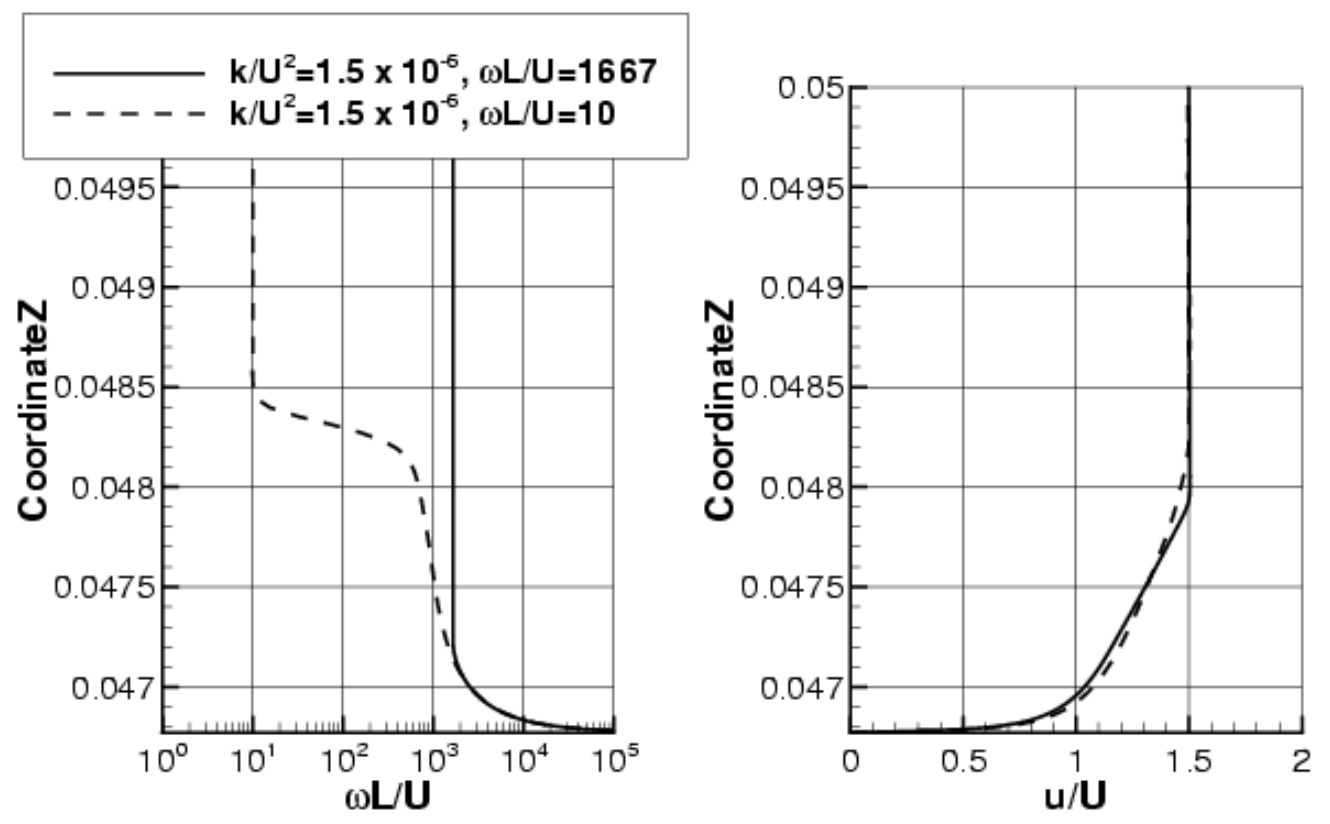

Figure 2. Effect of ambient $\omega$ on the boundary layer of a NACA 0012 airfoil, with $R e_{c}=10^{7}$

Figure 3 shows a solution of a multi-element airfoil at $R e_{c}=9 \times 10^{6}$, with the S-A model and different ambient turbulence levels. As $\tilde{\nu} / \nu$ is increased, the contamination in the solution gets progressively worse; note, however, that the solution degradation only starts to become noticeable at very high levels, greater than 500 or so (the low ambient value of $\tilde{\nu} / \nu=5$ is close to the recommended value of 3, and could be considered as "standard"). In other words, the acceptable range for $\tilde{\nu} / \nu$ is very wide. However, note that this range shrinks with lower Reynolds numbers.

Figure 4 displays the eddy viscosity, comparing a standard ambient value of $\tilde{\nu}_{F S} / \nu=5$ with a high value of $\tilde{\nu}_{F S} / \nu=5000$. Using the ambient turbulent value of 5 makes $\tilde{\nu} / \nu$ visible on a scale of hundreds only in the free shear layers, and clearly originating near the walls. In contrast, the solution with excessive ambient $\tilde{\nu}$ has a high plateau away from the airfoil. The S-A destruction term activates in response to the high plateau level and strongly reduces $\tilde{\nu} / \nu$, from 5000 gradually towards 0 near the wall. It is still excessive, of course, and the eddy-viscosity fields are grossly different, particularly in the free shear layers. Here we discuss the reasons why the excessively high $\tilde{\nu}$ degrades the solution. The approximate destruction equation is: $u \cdot \nabla(1 / \tilde{\nu})=2 c_{w 1} / d^{2}$, where $d$ is the distance to the wall (because in irrotational regions $r \gg 1$, so that $f_{w}=c_{w 3}=2$ ). For a streamline directly approaching the airfoil without deceleration, its solution is

$$
\frac{1}{\tilde{\nu}}=\frac{1}{\tilde{\nu}_{F S}}+\frac{2 c_{w 1}}{U d}
$$

which limits $\tilde{\nu}$ from above with $U d / 2 c_{w 1}$ (recall that $c_{w 1}=3.2$ ). This explains why the plateau at $\tilde{\nu}=5.5 \times 10^{-4} U L$ starts dropping near $d=0.05$, ahead of the slat, in Figure 4b. The drop is then faster than in Equation 10, because the local velocity $u$ is rapidly dropping below $U$. For streamlines that flow past the airfoil, the velocity is closer to $U$, but the destruction effect is cumulative, and a kind of super-boundary layer forms. For a gap flow that would be entered by fluid without deceleration, the rough upper bound $U d / 2 c_{w 1}$ gives a gap Reynolds number no lower than $4 c_{w 1}$, or 12.8; typically it is well above 12.8 thanks to destruction accumulated along the airfoil. In figure $4 \mathrm{~b}$, the eddy viscosity in the flap gap does not exceed $\tilde{\nu} / \nu=750$; compare with $\tilde{\nu}_{F S} / \nu=5000$ and $U d / 2 c_{w 1} / \nu \approx 7000$. The gap Reynolds number is about 180 . With the normal inflow value in figure $4 \mathrm{a}$, the gap Reynolds number is just 375 if based on the peak eddy viscosity, which propagates from the recirculation region in the flap cove. These two gap values for the different ambient levels are not hugely different, showing how the S-A model corrected the excessive inflow value to a good extent, for the gap. It also did rather well for the stagnation point. These are favorable consequences of a term which was calibrated for destruction in the logarithmic layer. However, the destruction term offers the free shear layers little protection: the peak eddy viscosity in the slat wake is artificially raised from about 2000 to 3000 when freestream $\tilde{\nu}_{F S} / \nu$ goes from 5 to 5000 .

Figure 5 illustrates the end effect of the variations on the lift coefficient. Again, $\tilde{\nu} / \nu$ needs to reach 500 , or about 

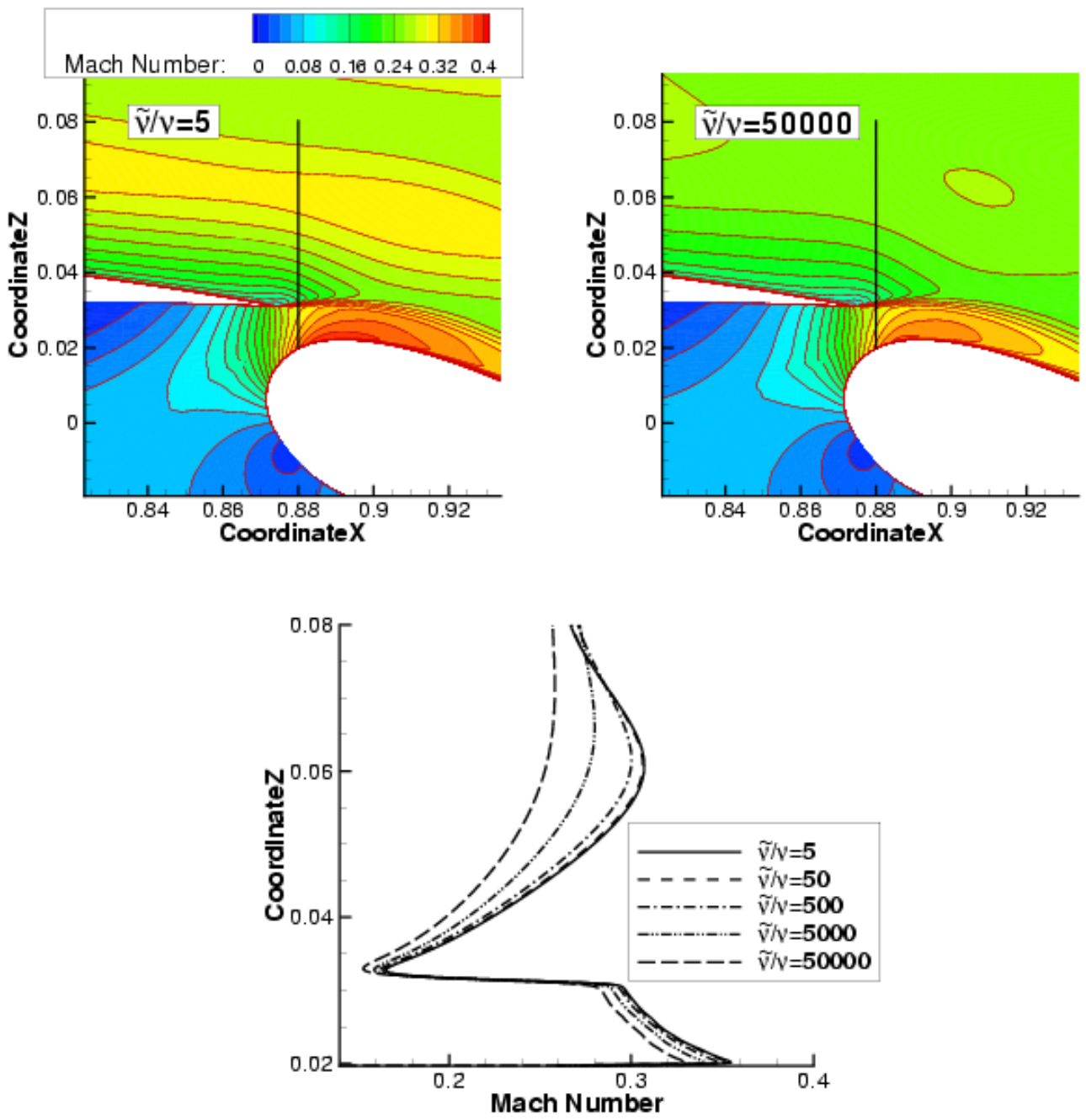

Figure 3. Multi-element airfoil flow fields using $\mathbf{S}-\mathbf{A}, \mathbf{M}=\mathbf{0 . 2}, \operatorname{Re} e_{c}=9$ million, showing (a) Mach contours near the flap gap for $\tilde{\nu} / \nu=5$, (b) same for $\tilde{\nu} / \nu=50000$, and (c) profiles of Mach number along $x / c=0.88$, for $\tilde{\nu} / \nu=5$ to 5000 in factors of 10.

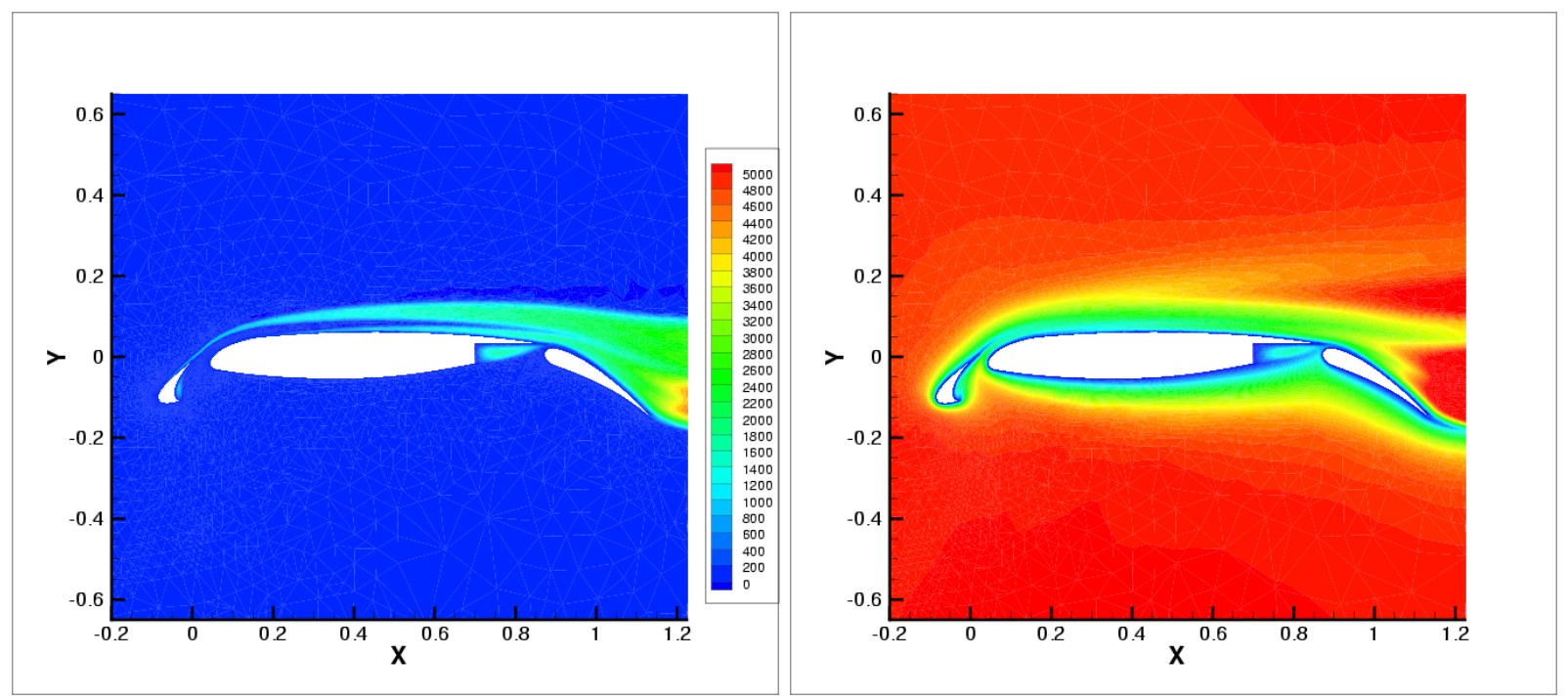

Figure 4. Multi-element eddy-viscosity fields $\nu_{t} / \nu$ using $\mathbf{S}-\mathbf{A}, \mathbf{M}=\mathbf{0 . 2}, R e_{c}=9$ million, showing (a) inflow $\tilde{\nu}_{F S} / \nu=5$; (b) same for $\tilde{\nu}_{F S} / \nu=5000$. Courtesy S. Allmaras. 
$R e_{c} / 2 \times 10^{4}$, before the effect on lift is appreciable (3\% with $\tilde{\nu} / \nu=500$, and $12 \%$ with $\left.\tilde{\nu} / \nu=5000\right)$. The estimate given earlier was about $R e_{c} / 10^{4}$, therefore quite close.

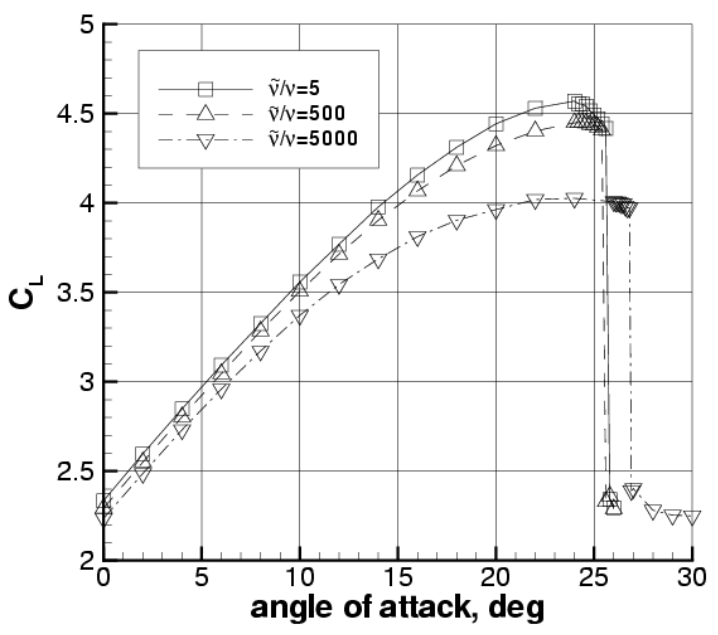

Figure 5. Lift coefficient of McDonnell-Douglas three-element airfoil with a range of values for ambient eddy viscosity. Chord Reynolds number $9 \times 10^{6}$. Courtesy S. Allmaras.

Turning our attention to the leading edge, we find that the values of $\delta / L$ are quite Reynolds-number dependent since the laminar thickness is of order $\sqrt{\nu r / U}$ with $r$ the leading-edge radius, and the turbulent thickness is often not much larger. However, the constraint on $\varepsilon$ or $\omega$ will be more severe if based on the thicker regions, where $\delta$ is a few percent of chord. Free-shear regions such as the slat wake have similar if not larger thicknesses, and smaller velocity differences, which weakens their resistance. The merger of the slat wake with the main-element wake has much influence on the flow at some angles of attack, and therefore this wake cannot be "sacrificed." This is not to imply that any of the present models approach perfection in predicting this merger; only that the solutions should be consistent between different codes and users.

\section{Recommended Values}

The considerations above lead to going-in upper bounds for the ambient primary-variable values $k \approx 1 \times 10^{-4} U^{2}$, $\varepsilon \approx 4.5 \times 10^{-5} U^{3} / c, \omega \approx 5 U / c$. Here, $c=L$ is the airfoil chord. Use of these upper bounds implies $\nu_{t} \approx 2 \times 10^{-5} U c$, which is small enough for flap gaps and well-developed boundary layers, but possibly too large for the leading-edge boundary layers. This may not strongly influence the rest of the flow, because the acceleration weakens the memory of the boundary layer. Still, some applications such as laminar-flow control and icing are dependent on high accuracy in the leading-edge region. This suggests lowering the allowable $k$, to say, $k \approx 1 \times 10^{-6} U^{2}$, while preserving $\omega \approx 5 U / c$. This gives an upper bound on $\varepsilon$ of $\approx 4.5 \times 10^{-7} U^{3} / c$, and $\nu_{t} \approx 2 \times 10^{-7} U c$.

Note that this ambient value of $\omega$ (or $\varepsilon / C_{\mu} k$ ) is unreachable if free decay takes place. An accurate solution for free decay sets the value of $k / \varepsilon$ in the neighborhood of $100 c / U$ for a $50 c$ domain, or $\omega \approx 0.1 U / c$. Such a low value is not required by comparison with the boundary layer (fig. 2), and is undesirable for the following reason: the SST model can encounter a particular problem when the ambient value of $\nu_{t} / \nu$ is large and $\omega$ is set too small. The SST limiter chooses the maximum of $a_{1} \omega$ and $\Omega F_{2}$ ( $\Omega$ the vorticity). Outside the boundary layer, $\Omega \rightarrow 0$ and $F_{2} \rightarrow 0$, and it is the intention that the model choose $a_{1} \omega$ in this region. However, if $\omega$ is too small and residual vorticity is present, the two terms compete and the result can be an irregular eddy-viscosity distribution, as shown in Fig. 6. The main problem with this erratic behavior is that convergence can stall. Some implementations of the SST model use the strain rate instead of $\Omega$, which is neutral in thin shear flows, but this is not the reference formula. In our tests with the NACA 0012 airfoil, values of $\nu_{t} / \nu>1$ and $\omega c / U<3$ or so caused such problems. Imposing floor values are quite helpful in dealing with this problem. Figure 3 suggests that values of $\omega c / U$ up to 100 would be more than safe, so that the useable range is not too narrow.

To summarize, the following ambient freestream turbulence values (in the region near the body) are recommended for general use for most typical external aerodynamic applications with two-equation turbulence models: $k / U^{2}=$ $1 \times 10^{-6}$ (or $T u$ near $\approx 0.1 \%$ ), $\varepsilon c / U^{3}=4.5 \times 10^{-7}, \omega c / U=5$. These values yield an ambient eddy viscosity ratio 


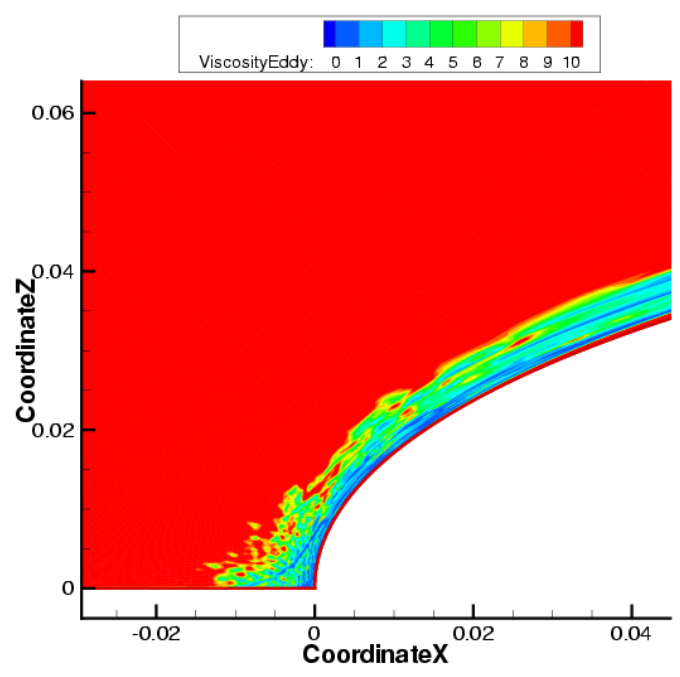

Figure 6. Example of poor solution behavior of SST model outside the boundary layer edge, due to too-low ambient $\omega L / U=0.2$, $\nu_{t} / \nu=12.5$, NACA 0012 airfoil, $\alpha=0^{\circ}, R e_{c}=10^{7}$.

of $\nu_{t} / \nu \approx 2 \times 10^{-7} \times \operatorname{Re}_{c}$. Thus, for example, for $R e_{c}=10$ million, $\nu_{t} / \nu \approx 2$; and for $R e_{c}=1$ million, $\nu_{t} / \nu \approx 0.2$. Again, these ambient levels are not achievable if the turbulence variables are allowed to decay naturally according to the turbulence equations. One easy way to achieve these ambient levels in practice is to impose them as both inflow and floor values.

For S-A, the values $\tilde{\nu} / \nu=3$ and 5 are well-placed for fully-turbulent behavior, with 3 somewhat preferable at the lowest Reynolds numbers, since it gives $\nu_{t} / \nu \approx 0.2$, well below 1 . Higher values may slightly help convergence at high Reynolds numbers by smoothing out the edges of turbulent regions, but not enough to motivate giving up the simple recommendation.

Figure 7 explores the S-A flow field for a multi-element configuration using $\tilde{\nu}_{F S} / \nu=5$, with emphasis on the stagnation regions. An exponential scale is used in this figure for eddy viscosity. It is quite smooth on this grid, although with a little noise in the 5-to-10 range. Although not shown, a computation on a coarser grid (about 1/4 the number of points) was not smooth for the low values of $\tilde{\nu}$ up to about 50, and it also had significant negative excursions of $\tilde{\nu}$ in the flap gap, traced to irregularities in the adaptive unstructured grid. The fine grid has negative values only very far downstream (and in this code, when $\tilde{\nu}<0, \nu_{t}$ is set to 0 ). Multi-block structured grids generate their own disruptions, but they appear to be milder, and confined to block corners and small regions with highly stretched and distorted cells. There is no evidence here that the imperfect solution is harming the "physics" of the model, but a blanket statement can certainly not be made. For instance, contact between unphysical "hot spots" and the flap upper-surface boundary layer could precipitate transition in a grid-dependent manner.

Also striking in this computation is the fact that the model does not depart from laminar for quite some distance in some of the boundary layers. For instance, the lower stagnation point on the slat is near $x=-0.035$, and appreciable eddy viscosity does not grow until the adverse pressure gradient at $x=-0.085$. This supports an earlier comment about the boundary-layer thicknesses in laminar and turbulent flow in stagnation regions being close (even at a high chord Reynolds number). The boundary layer is vigorously thinned out by the acceleration, yet the model remains poised to grow turbulent values, because the ambient value was chosen for that. The flap lower-surface boundary layer, similarly, is free of significant turbulence within the frame shown; it grows very gradually, to reach 27 at the trailing edge. The Turbulence Index $i_{t}$ (ref. 4 ) is about 0.5 at the stagnation points, but rises to 1 rapidly.

Figure 8 is a similar figure for the SST model; the left two frames can be directly compared with those in Figure 7. The three turbulence variables are shown in exponential scales again, with the darkest blue for the ambient values. The recommended ambient values (also used as floor values) appear very effective, in that they are smaller than the boundary-layer values almost everywhere along the wall, yet transition is occuring reliably. Note mild differences between the S-A and SST models in the slat cove: the peak eddy-viscosity values are 1650 and 1800, respectively, and the SST recirculation bubble is accordingly wider. Conversely, the slat-upper-surface boundary layer near $x=-0.025$, S-A peaks at 314 and SST at 277, and the S-A boundary layer is marginally thicker. Similar runs with the chord Reynolds number ranging from $10^{6}$ to $10^{8}$ were all successful. 

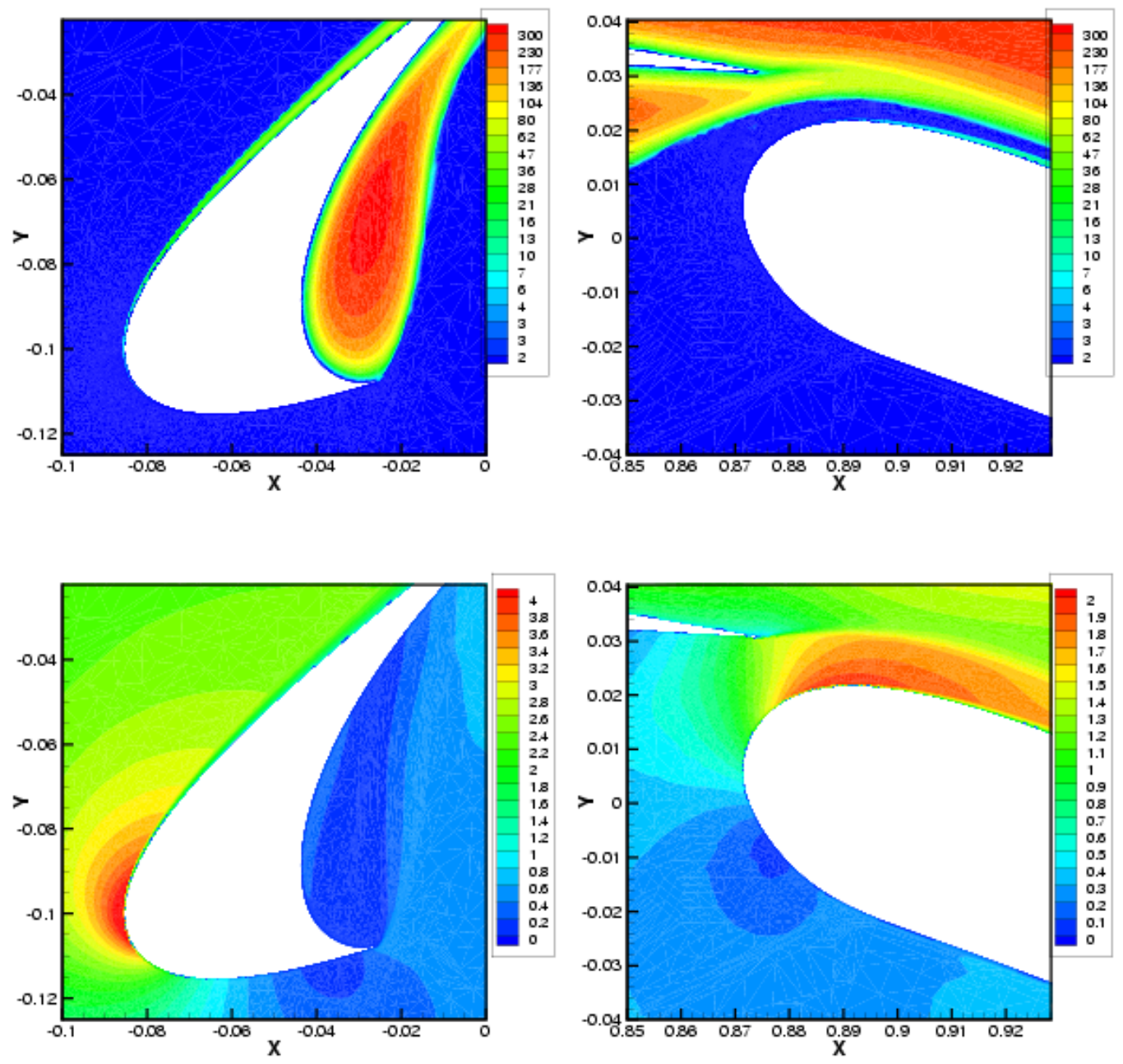

Figure 7. Detail of S-A solution, McDonnell-Douglas three-element airfoil. Upper frames, eddy viscosity; lower frames, velocity magnitude. Chord Reynolds number $9 \times 10^{6}, \alpha=24^{\circ}, \tilde{\nu}_{F S} / \nu=5$. Unstructured grid, courtesy S. Allmaras. 

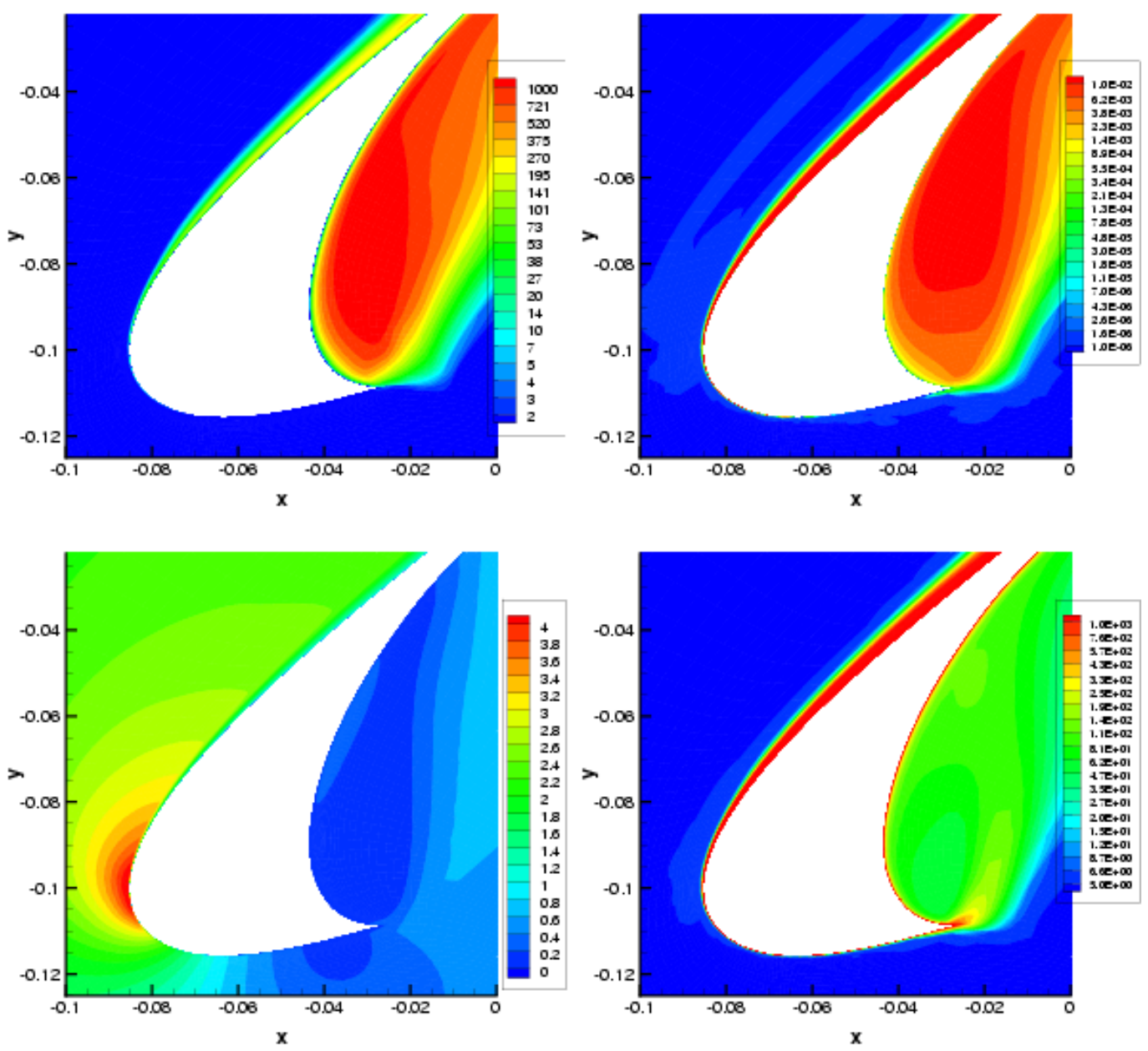

Figure 8. Detail of SST solution, McDonnell-Douglas three-element airfoil. Upper left, eddy viscosity $\nu_{t} / \nu$; upper right, $k_{F S} / U^{2}$; lower left, velocity magnitude; lower right, $\omega c / U$. $R e_{c}=9 \times 10^{6}, \alpha=19^{\circ}, k_{F S} / U^{2}=1 \times 10^{-6}, \omega_{F S} c / U=5$, with floor values. 
Finally, Fig. 9 demonstrates how use of the recommended ambient values in the SST model can reduce the griddependence of apparent transition location for an airfoil computation. In Rumsey, ${ }^{1}$ the grid dependence was first demonstrated for typically-used freestream turbulence levels, but a satisfactory explanation and cure were not found. Fig. 9(a) shows the sort of grid-dependent transition seen. Although relatively small, this grid-dependence could conceivably impact conclusions drawn from grid sensitivity studies, for example. In Fig. 9(b) we find that by applying the currently recommended freestream levels, and limiting via floor values (at the same levels), the grid-dependence in transition location is largely eliminated.

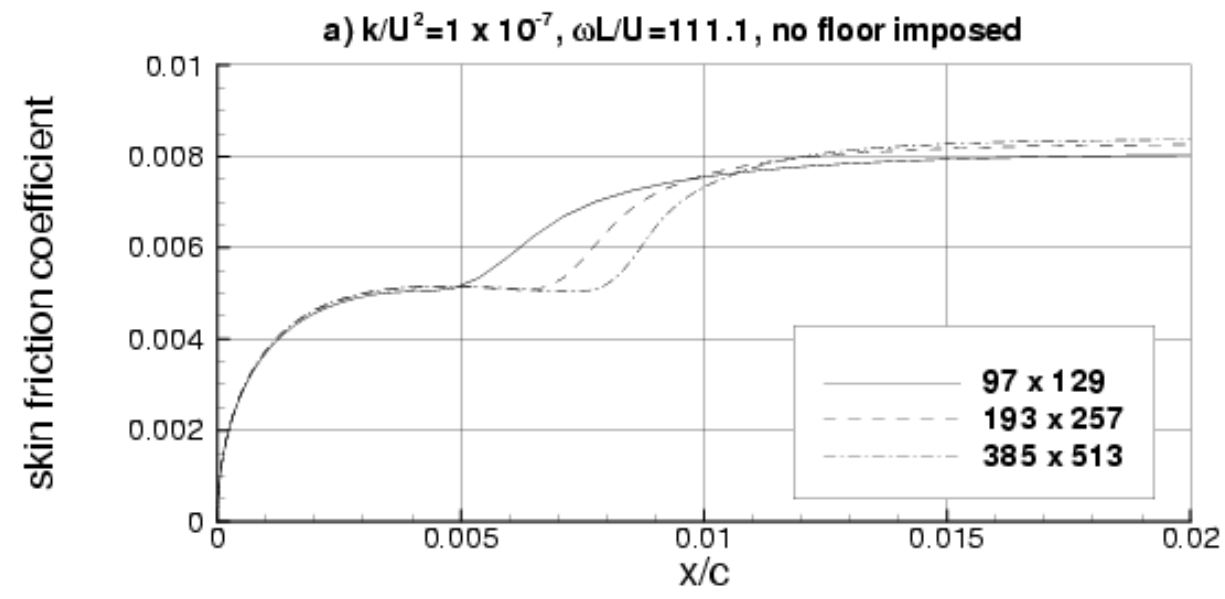

b) $k / U^{2}=1 \times 10^{-6}, \omega L / U=5$, floor imposed

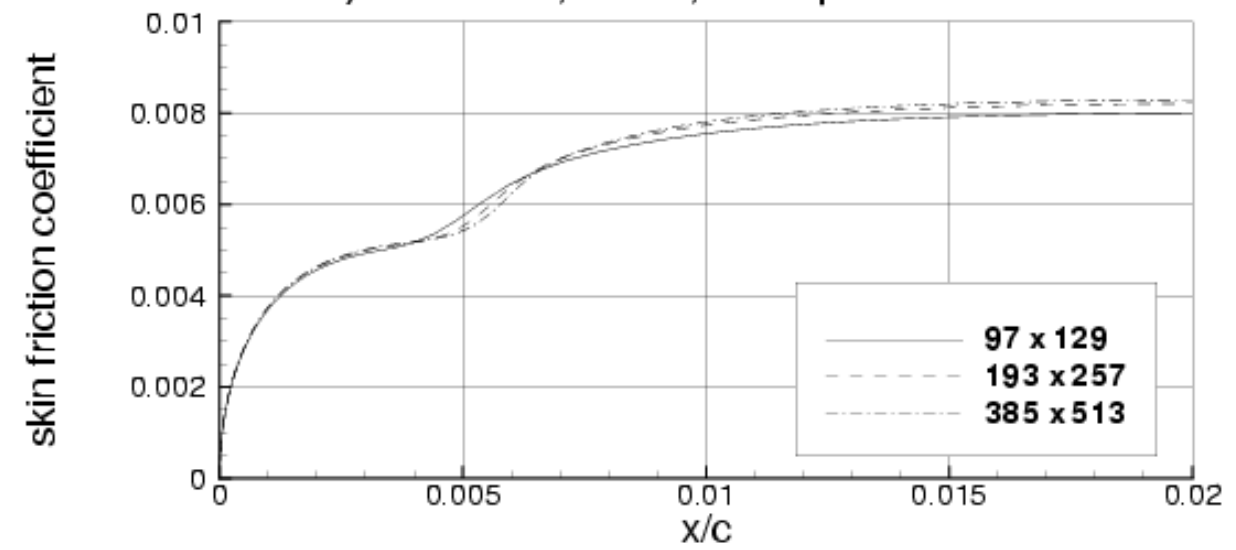

Figure 9. Skin friction coefficient near leading edge of NACA 0012 airfoil at $R e_{c}=10^{7}$, with SST model; (a) grid-dependent solution from Rumsey $^{1}$ using typical freestream turbulence levels and no floor values; (b) solution using recommended freestream turbulence levels and floor values.

\section{Summary}

Inflow conditions for CFD with simple turbulence models in large domains were studied from theoretical and pragmatic points of view, with the following outcomes: (1) simplistic and erroneous conceptions in the community were discussed; (2) rates of free decay for widely-used two-equation model levels were shown to be under-resolved on typical grids; (3) excessively high values of eddy viscosity were demonstrated to contaminate the flow field in non-turbulent regions; (4) the use of floor values, which greatly facilitate the control of the ambient levels in twoequation models, was advocated; (5) rational lower and upper bounds for these ambient values were provided; and (6) specific ambient turbulence values were recommended as safe starting points, located within rather wide intervals of low sensitivity. The practice of floor values, which conflicts with the two fundamental turbulence equations, was supported by theoretical considerations on the relevant range of turbulent motions. Thanks to having a single equation, the Spalart-Allmaras model readily provides an equivalent level of control, plus the ability to steer the boundary layers 
to be laminar or turbulent, without any special measures. If accepted, the present recommendations will spread in CFD codes, lowering the user's burden and re-work, improving convergence and consistency between different users, and improving the accuracy of CFD solutions by removing unseen contaminations and helping users employ the models precisely as their authors intended.

Acknowledgements. The authors thank Drs. M. Strelets and R. Langtry for valuable discussions, and Dr. S. Allmaras for supporting calculations and discussions.

\section{References}

\footnotetext{
${ }^{1}$ Rumsey, C. L., "Apparent transition behavior of widely-used turbulence models," AIAA-2006-3906. Revised version subm. to Int. J. Heat and Fluid Flow.

${ }^{2}$ Goldberg, U., Peroomian, O., Chakravarthy, S., and Sekar, B., "Validation of CFD++ Code Capability for Supersonic Combuster Flowfields," AIAA Paper 97-3271, 1997.

${ }^{3}$ Menter, F. R., "Two-Equation Eddy-Viscosity Turbulence Models for Engineering Applications," AIAA Journal, Vol. 32, No. 8, 1994, pp. $1598-1605$.

${ }^{4}$ Spalart, P. R., and Allmaras, S. R., “A One-Equation Turbulence Model for Aerodynamic Flows," La Recherche Aerospatiale, No. 1, 1994, pp. 5-21, also AIAA Paper 92-0439, January 1992.

${ }^{5}$ Baldwin, B. S. and Barth, T. J., “A One-Equation Turbulence Transport Model for High Reynolds Number Wall-Bounded Flows,” NASA TM-102847, August 1990.

${ }^{6}$ Abdol-Hamid, K. S., Lakshmanan, B., and Carlson, J. R., “Application of Navier-Stokes Code PAB3D with $k-\varepsilon$ Turbulence Model to Attached and Separated Flows," NASA TP-3480, January 1995.

${ }^{7}$ Menter, F. R., "Influence of Freestream Values on $k$ - $\omega$ Turbulence Model Predictions," AIAA Journal, Vol. 30, No. 6, 1992, pp. $1657-1659$.

${ }^{8}$ Menter, F. R., "Eddy Viscosity Transport Equations and their Relation to the $k$ - $\varepsilon$ Model," J. Fluids Eng., Vol. 119, No. 12, 1997, pp. 876-884.

${ }^{9}$ Wilcox, D. C., Turbulence Modeling for CFD, 1st ed., DCW Industries, La Canada, 1994.

${ }^{10}$ Kalitzen, G., "Application of the $v^{2}-f$ Turbulence Model to Transonic Flows," AIAA Paper 99-3780, June-July 1999.

${ }^{11}$ Cazalbou, J. B., Spalart, P. R., and Bradshaw, P., "On the Behavior of Two-Equation Models at the Edge of a Turbulent Region.” Phys. Fluids A, 6 (5), 1994, 1797-1804.
} 